\title{
Pseudo Asymptotic Behavior of Mild Solution for Nonautonomous Integrodifferential Equations with Nondense Domain
}

\author{
Zhinan Xia \\ Department of Applied Mathematics, Zhejiang University of Technology, Hangzhou, \\ Zhejiang 310023, China \\ Correspondence should be addressed to Zhinan Xia; xiazn299@zjut.edu.cn
}

Received 6 April 2014; Accepted 15 July 2014; Published 11 August 2014

Academic Editor: Bogdan Sasu

Copyright ( 2014 Zhinan Xia. This is an open access article distributed under the Creative Commons Attribution License, which permits unrestricted use, distribution, and reproduction in any medium, provided the original work is properly cited.

By the weighted ergodic function based on the measure theory, we study pseudo asymptotic behavior of mild solution for nonautonomous integrodifferential equations with nondense domain. The existence and uniqueness of $\mu$-pseudo antiperiodic ( $\mu$ pseudo periodic, $\mu$-pseudo almost periodic, and $\mu$-pseudo automorphic) solution are investigated. Some interesting examples are presented to illustrate the main findings.

\section{Introduction}

The study of pseudo asymptotic behavior of solution is one of the most interesting and important topics in the qualitative theory of differential equations. Much work has been done to investigate the existence of pseudo antiperiodic, pseudo periodic, pseudo almost periodic, and pseudo almost automorphic solution for differential equations [1-5]. Recently, Blot et al. $[6,7]$ used the results of the measure theory to establish $\mu$-ergodic and introduce the new concepts of $\mu$ pseudo almost periodic and $\mu$-pseudo almost automorphic function, which are more general than pseudo almost periodic and pseudo almost automorphic function, respectively. They developed some results like completeness and composition theorems to investigate differential equations in Banach space.

Integrodifferential equations play a crucial role in qualitative theory of differential equations due to their application to physics, engineering, biology, and other subjects. This type of equations has received much attention in recent years and the general asymptotic behavior of solution is at present an active source of research.
In this paper, we study pseudo asymptotic behavior of solution to the following nonautonomous integrodifferential equations with nondense domain:

$$
\begin{gathered}
u^{\prime}(t)=A(t) u(t)+f(t, u(t),(K u)(t)), \quad t \in \mathbb{R}, \\
(K u)(t)=\int_{-\infty}^{t} k(t-s) g(s, u(s)) d s,
\end{gathered}
$$

where the linear operators $A(t): D(A(t)) \subset X \rightarrow X$ have a domain $D(A(t))$ not necessarily dense in Banach space $X$ and satisfy "Acquistapace-Terreni" conditions and $f, g$, and $k$ are continuous functions.

Some recent contributions on almost periodic, almost automorphic, pseudo almost periodic, and pseudo almost automorphic solution to integrodifferential equations of the form (1) in the case $A(t)=A$ are constant $[4,8-13]$. However, for the nonautonomous case, that is, (1), the study of pseudo asymptotic behavior of solution is rare [14]. In this paper, we will make use of the so-called "AcquistapaceTerreni" conditions associated with exponential dichotomy, fixed point theorem to derive some sufficient conditions to 
the existence and uniqueness of $\mu$-pseudo antiperiodic ( $\mu$ pseudo periodic, $\mu$-pseudo almost periodic, and $\mu$-pseudo almost automorphic) mild solution to (1).

The paper is organized as follows. In Section 2, we recall some fundamental results about the notion of $\mathscr{P}$-class of functions including composition theorem. Section 3 is devoted to pseudo asymptotic behavior of mild solution to nonautonomous integrodifferential equations with nondense domain. In Section 4, we provide some examples to illustrate our main results.

\section{Preliminaries and Basic Results}

Let $(X,\|\cdot\|)$ and $(Y,\|\cdot\|)$ be two Banach spaces and $\mathbb{N}, \mathbb{Z}$, $\mathbb{R}$, and $\mathbb{C}$ stand for the set of natural numbers, integers, real numbers, and complex numbers, respectively. For $A$ being a linear operator on $X, D(A), \rho(A), R(\lambda, A)$, and $\sigma(A)$ stand for the domain, the resolvent set, the resolvent, and spectrum of $A$. In order to facilitate the discussion below, we further introduce the following notations:

(i) $B C(\mathbb{R}, X)($ resp., $B C(\mathbb{R} \times Y, X))$ : the Banach space of bounded continuous functions from $\mathbb{R}$ to $X$ (resp., from $\mathbb{R} \times Y$ to $X)$ with the supremum norm;

(ii) $C(\mathbb{R}, X)$ (resp. $C(\mathbb{R} \times Y, X)$ ): the set of continuous functions from $\mathbb{R}$ to $X$ (resp., from $\mathbb{R} \times Y$ to $X$ );

(iii) $L(X, Y)$ : the Banach space of bounded linear operators from $X$ to $Y$ endowed with the operator topology; in particular, we write $L(X)$ when $X=Y$;

(iv) $L^{p}(\mathbb{R}, X)$ : the space of all classes of equivalence (with respect to the equality almost everywhere on $\mathbb{R}$ ) of measurable functions $f: \mathbb{R} \rightarrow X$ such that $\|f\| \epsilon$ $L^{p}(\mathbb{R}, \mathbb{R})$.

\subsection{Evolution Family and Exponential Dichotomy}

Definition 1. A family of bounded linear operators $(U(t, s))_{t \geq s}$ on a Banach space $X$ is called a strong continuous evolution family if

(i) $U(t, r) U(r, s)=U(t, s)$ and $U(s, s)=I$ for all $t \geq r \geq s$ and $t, r, s \in \mathbb{R}$;

(ii) the map $(t, s) \rightarrow U(t, s) x$ is continuous for all $x \in$ $X, t \geq s$ and $t, s \in \mathbb{R}$.

Definition 2. An evolution family $(U(t, s))_{t \geq s}$ on a Banach space $X$ is called hyperbolic (or has an exponential dichotomy) if there exist projections $P(t), t \in \mathbb{R}$, uniformly bounded and strongly continuous in $t$ and constants $c>0$, $\delta>0$ such that

(i) $U(t, s) P(s)=P(t) U(t, s)$ for $t \geq s$ and $t, s \in \mathbb{R}$,

(ii) the restriction $U_{\mathrm{Q}}(t, s): Q(s) X \rightarrow Q(t) X$ of $U(t, s)$ is invertible for $t \geq s$ (and set $\left.U_{Q}(s, t):=U(t, s)^{-1}\right)$, (iii)

$$
\begin{gathered}
\|U(t, s) P(s)\| \leq M e^{-\delta(t-s)}, \\
\left\|U_{Q}(s, t) Q(t)\right\| \leq M e^{-\delta(t-s)},
\end{gathered}
$$

for $t \geq s$ and $t, s \in \mathbb{R}$. Here and next we set $Q:=I-P$.

Remark 3. Exponential dichotomy is a classical concept in the study of long-time behaviour of evolution equations. If $P(t)=$ $I$ for $t \in \mathbb{R}$, then $(U(t, s))_{t \geq s}$ is exponentially stable. One can see [15-17] for more details.

If $(U(t, s))_{t \geq s}$ is hyperbolic, then

$$
\Gamma(t, s):= \begin{cases}U(t, s) P(s), & t \geq s, t, s \in \mathbb{R}, \\ -U_{Q}(t, s) Q(s), & t<s, t, s \in \mathbb{R},\end{cases}
$$

is called Green's function corresponding to $(U(t, s))_{t \geq s}, P(\cdot)$ and

$$
\|\Gamma(t, s)\| \leq \begin{cases}M e^{-\delta(t-s)}, & t \geq s, t, s \in \mathbb{R}, \\ M e^{-\delta(s-t)}, & t<s, t, s \in \mathbb{R} .\end{cases}
$$

2.2. $\mu$-Ergodic and Functions by Measure Theory. $\mathscr{B}$ denotes the Lebesgue $\sigma$-field of $\mathbb{R}$ and $\mathscr{M}$ stands for the set of all positive measures $\mu$ on $\mathscr{B}$ satisfying $\mu(\mathbb{R})=\infty$ and $\mu([a, b])<$ $\infty$ for all $a, b \in \mathbb{R}(a \leq b)$. We formulate the following hypothesis.

$\left(H_{0}\right)$ For all $\tau \in \mathbb{R}$, there exist $\beta>0$ and a bounded interval I such that

$\mu(\{a+\tau, a \in A\}) \leq \beta \mu(A) \quad$ if $A \in \mathscr{B}$ satisfies $A \cap I=\emptyset$.

Definition 4 (see [6]). Let $\mu \in \mathscr{M}$. A function $f \in B C(\mathbb{R}, X)$ is said to be $\mu$-ergodic if

$$
\lim _{T \rightarrow+\infty} \frac{1}{\mu([-T, T])} \int_{[-T, T]}\|f(t)\| d \mu(t)=0 .
$$

Denote by $\mathscr{E}(\mathbb{R}, X, \mu)$ the set of such functions.

Lemma 5 (see [6]). Let $\mu \in \mathscr{M}$ and satisfy $\left(H_{0}\right)$; then $\mathscr{E}(\mathbb{R}$, $X, \mu)$ is a translation invariant.

Definition 6. A function $f \in C(\mathbb{R}, X)$ is said to be antiperiodic if there exists a $\omega \in \mathbb{R} \backslash\{0\}$ with the property that $f(t+\omega)=-f(t)$ for all $t \in \mathbb{R}$. If there exists a less positive $\omega$ with this property, it is called the antiperiodic of $f$. The collection of those functions is denoted by $P_{\text {wap }}(\mathbb{R}, X)$.

Definition 7. A function $f \in C(\mathbb{R}, X)$ is said to be periodic if there exists a $\omega \in \mathbb{R} \backslash\{0\}$ with the property that $f(t+\omega)=$ $f(t)$ for all $t \in \mathbb{R}$. If there exists a less positive $\omega$ with this property, it is called the periodic of $f$. The collection of those $\omega$-periodic functions is denoted by $P_{\omega}(\mathbb{R}, X)$.

Definition 8 (see [18]). A function $f \in C(\mathbb{R}, X)$ is said to be almost periodic if for each $\varepsilon>0$, there exists an $l(\varepsilon)>0$, such that every interval $I$ of length $l(\varepsilon)$ contains a number $\tau$ with the property that $\|f(t+\tau)-f(t)\|<\varepsilon$ for all $t \in \mathbb{R}$. Denote by $A P(\mathbb{R}, X)$ the set of such functions. 
Definition 9 (see [19]). A function $f \in C(\mathbb{R}, X)$ is said to be almost automorphic if for every sequence of real numbers $\left(s_{n}^{\prime}\right)_{n \in \mathbb{N}}$, there exists a subsequence $\left(s_{n}\right)_{n \in \mathbb{N}}$ such that

$$
g(t):=\lim _{n \rightarrow \infty} f\left(t+s_{n}\right)
$$

is well defined for each $t \in \mathbb{R}$, and

$$
\lim _{n \rightarrow \infty} g\left(t-s_{n}\right)=f(t)
$$

for each $t \in \mathbb{R}$. Denote by $A A(\mathbb{R}, X)$ the set of such functions.

Next, we recall the $\mu$-class of functions by the measure theory.

Definition 10. Let $\mu \in \mathscr{M}$. A function $f \in C(\mathbb{R}, X)$ is said to be $\mu$-pseudo antiperiodic if it can be decomposed as $f=$ $g+\varphi$, where $g \in P_{\text {wap }}(\mathbb{R}, X)$ and $\varphi \in \mathscr{E}(\mathbb{R}, X, \mu)$. Denote by $P P_{\text {wap }}(\mathbb{R}, X, \mu)$ the collection of such functions.

Definition 11. Let $\mu \in \mathscr{M}$. A function $f \in C(\mathbb{R}, X)$ is said to be a $\mu$-pseudo periodic if it can be decomposed as $f=$ $g+\varphi$, where $g \in P_{\omega}(\mathbb{R}, X)$ and $\varphi \in \mathscr{E}(\mathbb{R}, X, \mu)$. Denote by $P P_{\omega}(\mathbb{R}, X, \mu)$ the collection of such functions.

Definition 12 (see [6]). Let $\mu \in \mathscr{M}$. A function $f \in C(\mathbb{R}, X)$ is said to be $\mu$-pseudo almost periodic if it can be decomposed as $f=g+\varphi$, where $g \in A P(\mathbb{R}, X)$ and $\varphi \in \mathscr{E}(\mathbb{R}, X, \mu)$. Denote by $P A P(\mathbb{R}, X, \mu)$ the collection of such functions.

Definition 13 (see [7]). Let $\mu \in \mathscr{M}$. A function $f \in C(\mathbb{R}, X)$ is said to be $\mu$-pseudo almost automorphic if it can be decomposed as $f=g+\varphi$, where $g \in A A(\mathbb{R}, X)$ and $\varphi \in \mathscr{E}(\mathbb{R}, X, \mu)$. Denote by $P A A(\mathbb{R}, X, \mu)$ the collection of such functions.

Remark 14. (i) If the measure $\mu$ is the Lebesgue measure, then $P P_{\text {wap }}(\mathbb{R}, X, \mu), P P_{\omega}(\mathbb{R}, X, \mu), P A P(\mathbb{R}, X, \mu)$, and $P A A(\mathbb{R}, X, \mu)$ are the following functions: pseudo antiperiodic $\left(P P_{\text {wap }}(\mathbb{R}, X)[5]\right)$, pseudo periodic $\left(P P_{\omega}(\mathbb{R}, X)[4]\right)$, pseudo almost periodic $(P A P(\mathbb{R}, X)[20])$, and pseudo almost automorphic $(P A A(\mathbb{R}, X)[21])$, respectively. One can see $[6,7,22]$ for more details.

(ii) Let $\rho(t)>0$ a.e. on $\mathbb{R}$ for the Lebesgue measure. $\mu$ denotes the positive measure defined by

$$
\mu(A)=\int_{A} \rho(t) d t \quad \text { for } A \in \mathscr{B},
$$

where $d t$ denotes the Lebesgue measure on $\mathbb{R}$; then $P P_{\text {wap }}(\mathbb{R}$, $X, \mu), P P_{\omega}(\mathbb{R}, X, \mu), P A P(\mathbb{R}, X, \mu)$, and $P A A(\mathbb{R}, X, \mu)$ are the weighted class of functions: weighted pseudo antiperiodic $\left(W P P_{\text {wap }}(\mathbb{R}, X)[1]\right)$, weighted pseudo periodic $\left(W P P_{\omega}(\mathbb{R}, X)\right.$ $[1])$, weighted pseudo almost periodic $(W P A P(\mathbb{R}, X)[3])$, and weighted pseudo almost automorphic (WPAA(R, X) [2]), respectively.
Let

$$
\begin{aligned}
& \mathscr{A}(\mathbb{R}, X)=\left\{P_{\text {wap }}(\mathbb{R}, X), P_{\omega}(\mathbb{R}, X), A P(\mathbb{R}, X), A A(\mathbb{R}, X)\right\} \\
& \mathscr{P}(\mathbb{R}, X, \mu)=\left\{P P_{\text {wap }}(\mathbb{R}, X, \mu), P P_{\omega}(\mathbb{R}, X, \mu),\right. \\
& P A P(\mathbb{R}, X, \mu), P A A(\mathbb{R}, X, \mu)\} .
\end{aligned}
$$

It is not difficult to see that $f \in \mathscr{P}(\mathbb{R}, X, \mu)$ if and only if it can be decomposed as $f=g+\varphi$, where $g \in \mathscr{A}(\mathbb{R}, X)$ and $\varphi \in \mathscr{E}(\mathbb{R}, X, \mu)$.

Definition 15. Let $\mu_{1}, \mu_{2} \in \mathscr{M} ; \mu_{1}$ is said to be equivalent to $\mu_{2}\left(\mu_{1} \sim \mu_{2}\right)$ if there exist constants $\alpha, \beta>0$ and a bounded interval $I$ (eventually $I=\emptyset$ ) such that

$$
\begin{aligned}
& \alpha \mu_{1}(A) \leq \mu_{2}(A) \leq \beta \mu_{1}(A) \\
& \text { for } A \in \mathscr{B} \text { satisfies } A \cap I=\emptyset .
\end{aligned}
$$

Similarly as the proof of $[6,7]$, we have the following results for the class of functions $\mathscr{P}(\mathbb{R}, X, \mu)$.

Lemma 16. Let $\mu \in M$; then the following properties hold:

(i) $f \pm g \in \mathscr{P}(\mathbb{R}, X, \mu)$ if $f, g \in \mathscr{P}(\mathbb{R}, X, \mu)$;

(ii) $\lambda f \in \mathscr{P}(\mathbb{R}, X, \mu)$ if $\lambda \in \mathbb{R}, f \in \mathscr{P}(\mathbb{R}, X, \mu)$;

(iii) $\mathscr{P}(\mathbb{R}, X, \mu)$ is a Banach space with the supremum norm $\|\cdot\|$

(iv) $P P_{\text {wap }}(\mathbb{R}, X, \mu) \subset P P_{\omega}(\mathbb{R}, X, \mu) \subset P A P(\mathbb{R}, X, \mu) \subset$ $P A A(\mathbb{R}, X, \mu) \subset B C(\mathbb{R}, X)$.

Lemma 17. Let $\mu_{1}, \mu_{1} \in \mathscr{M}$. If $\mu_{1} \sim \mu_{2}$, then $\mathscr{E}\left(\mathbb{R}, X, \mu_{1}\right)=$ $\mathscr{E}\left(\mathbb{R}, X, \mu_{2}\right), \mathscr{P}\left(\mathbb{R}, X, \mu_{1}\right)=\mathscr{P}\left(\mathbb{R}, X, \mu_{2}\right)$.

Theorem 18. Let $\mu \in \mathscr{M}, f \in \mathscr{P}(\mathbb{R} \times X \times X, X, \mu)$ and satisfy the following:

(i) $f$ is uniformly continuous on each compact set $K_{1} \times K_{2}$ in $X \times X$ with respect to the second and third variables $u, v$;

(ii) for all bounded subsets $E_{1}, E_{2}$ of $X, f$ is bounded on $\mathbb{R} \times E_{1} \times E_{2}$.

Then $f\left(\cdot, h_{1}(\cdot), h_{2}(\cdot)\right) \in \mathscr{P}(\mathbb{R}, X, \mu)$ if $h_{1}(\cdot), h_{2}(\cdot) \in \mathscr{P}(\mathbb{R}, X$, $\mu$ ).

Corollary 19. Let $\mu \in \mathscr{M}$ and $f \in \mathscr{P}(\mathbb{R} \times X \times X, X, \mu)$, and there exists a constant $L_{f}>0$ such that

$$
\begin{array}{r}
\left\|f\left(t, u_{1}, u_{2}\right)-f\left(t, v_{1}, v_{2}\right)\right\| \leq L_{f}\left(\left\|u_{1}-v_{1}\right\|+\left\|u_{2}-v_{2}\right\|\right), \\
t \in \mathbb{R}, \quad u_{1}, u_{2}, v_{1}, v_{2} \in X ;
\end{array}
$$

then $f\left(\cdot, h_{1}(\cdot), h_{2}(\cdot)\right) \in \mathscr{P}(\mathbb{R}, X, \mu)$ if $h_{1}(\cdot), h_{2}(\cdot) \in \mathscr{P}(\mathbb{R}, X, \mu)$. 


\section{Nonautonomous Integrodifferential Equations}

This section is devoted to pseudo asymptotic behavior of mild solution to (1). In this section, we make the following assumptions.

$\left(H_{1}\right)$ There exist constants $\lambda_{0} \geq 0, \theta \in(\pi / 2, \pi), L, \widetilde{M} \geq 0$, and $\beta, \gamma \in(0,1)$ with $\beta+\gamma>1$ such that

$\Sigma_{\theta} \cup\{0\} \subset \rho\left(A(t)-\lambda_{0}\right), \quad\left\|R\left(\lambda, A(t)-\lambda_{0}\right)\right\| \leq \frac{\widetilde{M}}{1+|\lambda|}$,

$$
\begin{aligned}
& \|\left(A(t)-\lambda_{0}\right) R\left(\lambda, A(t)-\lambda_{0}\right) \\
& \quad \times\left[R\left(\lambda_{0}, A(t)\right)-R\left(\lambda_{0}, A(s)\right)\right] \| \\
& \leq L|t-s|^{\beta}|\lambda|^{-\gamma}
\end{aligned}
$$

for $t, s \in \mathbb{R}, \Sigma_{\theta}=\{\lambda \in \mathbb{C} \backslash\{0\}:|\arg \lambda| \leq \theta\}$.

$\left(H_{2}\right)$ The evolution family $(U(t, s))_{t \geq s}$ generated by $A(t)$ has an exponential dichotomy with constants $M>0$ and $\delta>0$; dichotomy projections $P(t), t \in \mathbb{R}$; and Green's function $\Gamma(t, s)$.

$\left(H_{3}\right)$ Consider $k \in C\left(\mathbb{R}^{+}, \mathbb{R}\right)$ and $|k(t)| \leq C_{k} e^{-b t}$ for some positive constants $C_{k}, b$.

$\left(H_{4}\right)$ There exists a constant $L_{g}>0$ such that

$$
\|g(t, u)-g(t, v)\| \leq L_{g}\|u-v\|, \quad t \in \mathbb{R}, u, v \in X .
$$

$\left(H_{5}\right)$ There exists a constant $L_{f}>0$ such that

$$
\begin{array}{r}
\left\|f\left(t, u_{1}, u_{2}\right)-f\left(t, v_{1}, v_{2}\right)\right\| \leq L_{f}\left(\left\|u_{1}-v_{1}\right\|+\left\|u_{2}-v_{2}\right\|\right), \\
t \in \mathbb{R}, \quad u_{1}, u_{2}, v_{1}, v_{2} \in X .
\end{array}
$$

$\left(H_{6}\right) \mu \in \mathscr{M}$ and satisfies $\left(H_{0}\right)$.

Remark 20. $\left(H_{1}\right)$ is usually called "Acquistapace-Terreni" conditions, which was first introduced in [23] and widely used to study nonautonomous differential equations in $[16,17$, 23-25]. If $\left(H_{1}\right)$ holds, there exists a unique evolution family $(U(t, s))_{t \geq s}$ on $X$, which governs the homogeneous version of (1) $[24]$.

Before starting our main results, we recall the definition of the mild solution to (1).

Definition 21 (see [26]). A mild solution of (1) is a continuous function $u: \mathbb{R} \rightarrow X$ satisfying

$$
u(t)=U(t, s) u(s)+\int_{s}^{t} U(t, \sigma) f(\sigma, u(\sigma),(K u)(\sigma)) d \sigma
$$

for all $t \geq s, t, s \in \mathbb{R}$.
Lemma 22. Assume that $h \in \mathscr{E}(\mathbb{R}, X, \mu)$ and $\left(H_{1}\right),\left(H_{2}\right)$, and $\left(\mathrm{H}_{6}\right)$ hold; then

$$
\begin{aligned}
(\Pi h)(t):= & \int_{-\infty}^{t} U(t, \sigma) P(\sigma) h(\sigma) d \sigma \\
& -\int_{t}^{\infty} U_{Q}(t, \sigma) Q(\sigma) h(\sigma) d \sigma \in \mathscr{E}(\mathbb{R}, X, \mu) .
\end{aligned}
$$

Proof. First, we show that $\Pi h$ is well defined. In fact, if $h \epsilon$ $\mathscr{E}(\mathbb{R}, X, \mu)$, so $\|h\|<\infty$. By $(2)$,

$$
\begin{gathered}
\|U(t, \sigma) P(\sigma) h(\sigma)\| \leq M e^{-\delta(t-\sigma)}\|h(\sigma)\| \leq M e^{-\delta(t-\sigma)}\|h\|, \\
\left\|U_{Q}(t, \sigma) Q(\sigma) h(\sigma)\right\| \leq M e^{-\delta(\sigma-t)}\|h(\sigma)\| \leq M e^{-\delta(\sigma-t)}\|h\| ;
\end{gathered}
$$

it follows that $U(t, \sigma) P(\sigma) h(\sigma)$ is integrable over $(-\infty, t)$ and $U_{Q}(t, \sigma) Q(\sigma) h(\sigma)$ is integrable over $(t, \infty)$ for $t \in \mathbb{R}$.

Note that $\Pi h \in B C(\mathbb{R}, X)$. Next, we show that

$$
\lim _{T \rightarrow+\infty} \frac{1}{\mu([-T, T])} \int_{[-T, T]}\|(\Pi h)(t)\| d \mu(t)=0 .
$$

In fact, for $T>0$, by using Fubini's theorem, one has

$$
\begin{aligned}
& \frac{1}{\mu([-T, T])} \int_{[-T, T]}\|(\Pi h)(t)\| d \mu(t) \\
& \leq \frac{1}{\mu([-T, T])} \int_{[-T, T]} \int_{-\infty}^{t}\|U(t, \sigma) P(\sigma) h(\sigma)\| d \sigma d \mu(t) \\
& +\frac{1}{\mu([-T, T])} \\
& \times \int_{[-T, T]} \int_{t}^{\infty}\left\|U_{\mathrm{Q}}(t, \sigma) Q(\sigma) h(\sigma)\right\| d \sigma d \mu(t) \\
& \leq \frac{1}{\mu([-T, T])} \int_{[-T, T]} \int_{-\infty}^{t} M e^{-\delta(t-\sigma)}\|h(\sigma)\| d \sigma d \mu(t) \\
& +\frac{1}{\mu([-T, T])} \int_{[-T, T]} \int_{t}^{\infty} M e^{-\delta(\sigma-t)}\|h(\sigma)\| d \sigma d \mu(t) \\
& =\frac{1}{\mu([-T, T])} \int_{[-T, T]} \int_{0}^{\infty} M e^{-\delta \sigma}\|h(t-\sigma)\| d \sigma d \mu(t) \\
& +\frac{1}{\mu([-T, T])} \int_{[-T, T]} \int_{0}^{\infty} M e^{-\delta \sigma}\|h(t+\sigma)\| d \sigma d \mu(t) \\
& =\int_{0}^{\infty} M e^{-\delta \sigma} \Phi_{T}(\sigma) d \sigma+\int_{0}^{\infty} M e^{-\delta \sigma} \Psi_{T}(\sigma) d \sigma,
\end{aligned}
$$

where

$$
\begin{aligned}
& \Phi_{T}(\sigma):=\frac{1}{\mu([-T, T])} \int_{[-T, T]}\|h(t-\sigma)\| d \mu(t), \\
& \Psi_{T}(\sigma):=\frac{1}{\mu([-T, T])} \int_{[-T, T]}\|h(t+\sigma)\| d \mu(t) .
\end{aligned}
$$


Since $\mu \in \mathscr{M}$ and satisfies $\left(H_{0}\right)$, by Lemma 5 , it follows that $h(\cdot-\sigma), h(\cdot+\sigma) \in \mathscr{E}(\mathbb{R}, X, \mu)$ for each $\sigma \in \mathbb{R}$; hence, $\lim _{T \rightarrow \infty} \Phi_{T}(\sigma)=0$ and $\lim _{T \rightarrow \infty} \Psi_{T}(\sigma)=0$ for all $\sigma \in \mathbb{R}$. Since $\left\|\Phi_{T}\right\|<\infty$ and $\left\|\Psi_{T}\right\|<\infty$, then $\Pi h \in \mathscr{E}(\mathbb{R}, X, \mu)$ by using the Lebesgue dominated convergence theorem.

Lemma 23. Assume that $h \in \mathscr{P}(\mathbb{R}, X, \mu)$ and $\left(H_{3}\right)$ and $\left(H_{6}\right)$ hold; then

$$
(\Lambda h)(t)=\int_{-\infty}^{t} k(t-s) h(s) d s \in \mathscr{P}(\mathbb{R}, X, \mu) .
$$

Proof. Similarly as the proof of Lemma 22 , it is not difficult to see that $\Lambda h$ is well defined and $\Lambda h \in B C(\mathbb{R}, X)$.

(i) Note that $h \in P P_{\omega a p}(\mathbb{R}, X, \mu), \mu \in \mathscr{M}$.

Let $h=h_{1}+h_{2}$, where $h_{1} \in P_{\text {wap }}(\mathbb{R}, X)$ and $h_{2} \in \mathscr{E}(\mathbb{R}$, $X, \mu)$; then

$$
(\Lambda h)(t)=\int_{-\infty}^{t} k(t-s) h(s) d s:=\left(\Lambda_{1} h\right)(t)+\left(\Lambda_{2} h\right)(t),
$$

where

$$
\begin{aligned}
& \left(\Lambda_{1} h\right)(t)=\int_{-\infty}^{t} k(t-s) h_{1}(s) d s \\
& \left(\Lambda_{2} h\right)(t)=\int_{-\infty}^{t} k(t-s) h_{2}(s) d s .
\end{aligned}
$$

Note that $h_{1} \in P_{\text {wap }}(\mathbb{R}, X)$; then

$$
\begin{aligned}
\left(\Lambda_{1} h\right)(t+\omega) & =\int_{-\infty}^{t+\omega} k(t+\omega-s) h_{1}(s) d s \\
& =\int_{-\infty}^{t} k(t-s) h_{1}(s+\omega) d s=-\left(\Lambda_{1} h\right)(t)
\end{aligned}
$$

hence, $\Lambda_{1} h \in P_{\omega p}(\mathbb{R}, X)$.

By using Fubini's theorem, one has

$$
\begin{aligned}
& \frac{1}{\mu([-T, T])} \int_{[-T, T]} \int_{-\infty}^{t}\left\|k(t-s) h_{2}(s)\right\| d s d \mu(t) \\
& \quad \leq \frac{1}{\mu([-T, T])} \int_{[-T, T]} \int_{-\infty}^{t} C_{k} e^{-b(t-s)}\left\|h_{2}(s)\right\| d s d \mu(t) \\
& \quad \leq \int_{0}^{\infty} \frac{C_{k}}{\mu([-T, T])} \int_{[-T, T]}\left\|h_{2}(t-s)\right\| d \mu(t) d s .
\end{aligned}
$$

Since $h_{2} \in \mathscr{E}(\mathbb{R}, X, \mu)$, it follows that $h_{2}(\cdot-s) \in \mathscr{E}(\mathbb{R}, X, \mu)$ for each $s \in \mathbb{R}$ by Lemma 5; hence, $\Lambda_{2} h \in \mathscr{E}(\mathbb{R}, X, \mu)$ by using the Lebesgue dominated convergence theorem. Hence, $\Lambda h \in P P_{\text {wap }}(\mathbb{R}, X, \mu)$.

(ii) Note that $h \in P P_{\omega}(\mathbb{R}, X, \mu), \mu \in M$.
Let $h=h_{1}+h_{2}$, where $h_{1} \in P_{\omega}(\mathbb{R}, X)$ and $h_{2} \in \mathscr{E}(\mathbb{R}, X, \mu) ;$ then

$$
\begin{aligned}
\left(\Lambda_{1} h\right)(t+\omega) & =\int_{-\infty}^{t+\omega} k(t+\omega-s) h_{1}(s) d s \\
& =\int_{-\infty}^{t} k(t-s) h_{1}(s+\omega) d s=\left(\Lambda_{1} h\right)(t) ;
\end{aligned}
$$

hence, $\Lambda_{1} h \in P_{\omega}(\mathbb{R}, X)$. Since $\Lambda_{2} h \in \mathscr{E}(\mathbb{R}, X, \mu)$ by (i), hence, $\Lambda h \in P P_{\omega}(\mathbb{R}, X, \mu)$.

(iii) Note that $h \in P A P(\mathbb{R}, X, \mu), \mu \in \mathscr{M}$.

By [27], $\Lambda_{1} h \in A P(\mathbb{R}, X)$. Since $\Lambda_{2} h \in \mathscr{E}(\mathbb{R}, X, \mu)$ by (i), hence, $\Lambda h \in P A P(\mathbb{R}, X, \mu)$.

(iv) Note that $h \in P A A(\mathbb{R}, X, \mu), \mu \in \mathscr{M}$.

Similarly as the proof of $[28], \Lambda_{1} h \in A A(\mathbb{R}, X)$. Since $\Lambda_{2} h \in \mathscr{E}(\mathbb{R}, X, \mu)$ by $(\mathrm{i}) ;$ hence, $\Lambda h \in P A A(\mathbb{R}, X, \mu)$.

3.1. Pseudo Almost Automorphic Perturbation. In this subsection, we investigated the existence and uniqueness of pseudo almost automorphic mild solution of (1).

First, we introduce the concept of bi-almost automorphic function.

Definition 24 (see [29]). A function $f \in C(\mathbb{R} \times \mathbb{R}, X)$ is called bi-almost automorphic if for every sequence of real numbers $\left(s_{n}^{\prime}\right)_{n \in \mathbb{N}}$, there exists a subsequence $\left(s_{n}\right)_{n \in \mathbb{N}}$ such that

$$
g(t, s):=\lim _{n \rightarrow \infty} f\left(t+s_{n}, s+s_{n}\right)
$$

is well defined for each $t, s \in \mathbb{R}$, and

$$
\lim _{n \rightarrow \infty} g\left(t-s_{n}, s-s_{n}\right)=f(t, s)
$$

for each $t, s \in \mathbb{R}$. The collection of all such functions will be denoted by $b A A(\mathbb{R} \times \mathbb{R}, X)$.

Now, we make the following assumptions:

$$
\begin{aligned}
& \left(H_{7_{1}}\right) \Gamma(t, s) x \in b A A(\mathbb{R} \times \mathbb{R}, X) \text { for each } x \in X ; \\
& \left(H_{8_{1}}\right) g \in P A A(\mathbb{R} \times X, X, \mu) \text { and } f \in P A A(\mathbb{R} \times X \times X, X, \mu) .
\end{aligned}
$$

Lemma 25 (see [25]). Assume that $h \in A A(\mathbb{R}, X),\left(H_{1}\right),\left(H_{2}\right)$, and $\left(H_{7_{1}}\right)$ hold; then

$$
\begin{aligned}
(\Pi h)(t)= & \int_{-\infty}^{t} U(t, \sigma) P(\sigma) h(\sigma) d \sigma \\
& -\int_{t}^{\infty} U_{Q}(t, \sigma) Q(\sigma) h(\sigma) d \sigma \in A A(\mathbb{R}, X) .
\end{aligned}
$$

Theorem 26. Suppose $\left(H_{1}\right)-\left(H_{6}\right),\left(H_{7_{1}}\right)$, and $\left(H_{8_{1}}\right)$ hold; if $2 M L_{f}\left(b+L_{g} C_{k}\right)<b \delta$, then (1) has a unique mild solution $u \in P A A(\mathbb{R}, X, \mu)$ such that

$$
\begin{aligned}
u(t)= & \int_{-\infty}^{t} U(t, \sigma) P(\sigma) f(\sigma, u(\sigma),(K u)(\sigma)) d \sigma \\
& -\int_{t}^{\infty} U_{Q}(t, \sigma) Q(\sigma) f(\sigma, u(\sigma),(K u)(\sigma)) d \sigma .
\end{aligned}
$$


Proof. First, we show that (1) admits a unique bounded solution given by (31), which is similar to the proof of [26, Theorem 3.3]. For $u \in P A A(\mathbb{R}, X, \mu)$, it is clear that $h(\cdot):=f(\cdot, u(\cdot),(K u)(\cdot)) \in P A A(\mathbb{R}, X, \mu)$ by Lemma 23 and Corollary 19; then $\|h\|<\infty$. By the definition of exponential dichotomy of $(U(t, s))_{t \geq s}$, it is not difficult to see that (31) is well defined for each $t \in \mathbb{R}$.

For all $t \geq s, t, s \in \mathbb{R}$,

$$
\begin{aligned}
u(s)= & \int_{-\infty}^{s} U(s, \sigma) P(\sigma) h(\sigma) d \sigma \\
& -\int_{s}^{\infty} U_{\mathrm{Q}}(s, \sigma) Q(\sigma) h(\sigma) d \sigma, \quad s \in \mathbb{R} ;
\end{aligned}
$$

then

$$
\begin{aligned}
U(t, s) & u(s) \\
= & \int_{-\infty}^{s} U(t, s) U(s, \sigma) P(\sigma) h(\sigma) d \sigma \\
& -\int_{s}^{\infty} U(t, s) U_{Q}(s, \sigma) Q(\sigma) h(\sigma) d \sigma \\
= & \int_{-\infty}^{s} U(t, \sigma) P(\sigma) h(\sigma) d \sigma \\
& -\int_{s}^{\infty} U_{Q}(t, \sigma) Q(\sigma) h(\sigma) d \sigma \\
= & \int_{-\infty}^{t} U(t, \sigma) P(\sigma) h(\sigma) d \sigma-\int_{s}^{t} U(t, \sigma) P(\sigma) h(\sigma) d \sigma \\
& -\int_{t}^{\infty} U_{Q}(t, \sigma) Q(\sigma) h(\sigma) d \sigma \\
& -\int_{s}^{t} U_{Q}(t, \sigma) Q(\sigma) h(\sigma) d \sigma \\
= & u(t)-\int_{s}^{t} U(t, \sigma) h(\sigma) d \sigma ;
\end{aligned}
$$

that is, $u$ is a mild solution of (1). To prove the uniqueness, let $v$ be another mild solution of (1); then

$$
v(t)=U(t, s) u(s)+\int_{s}^{t} U(t, \sigma) h(\sigma) d \sigma
$$

by the exponential dichotomy of $(U(t, s))_{t \geq s}$,

$$
P(t) v(t)=\int_{-\infty}^{t} U(t, \sigma) P(\sigma) h(\sigma) d \sigma, \quad t \in \mathbb{R} .
$$

Similarly,

$$
Q(t) v(t)=\int_{\infty}^{t} U_{Q}(t, \sigma) Q(\sigma) h(\sigma) d \sigma, \quad t \in \mathbb{R} .
$$

So,

$$
\begin{aligned}
v(t)= & P(t) v(t)+Q(t) v(t) \\
= & \int_{-\infty}^{t} U(t, \sigma) P(\sigma) h(\sigma) d \sigma \\
& -\int_{t}^{\infty} U_{Q}(t, \sigma) Q(\sigma) h(\sigma) d \sigma=u(t) .
\end{aligned}
$$

Next, define the operator $\mathscr{F}: P A A(\mathbb{R}, X, \mu) \rightarrow P A A(\mathbb{R}$, $X, \mu)$ by

$$
\begin{aligned}
(\mathscr{F} u)(t)= & \int_{-\infty}^{t} U(t, \sigma) P(\sigma) f(\sigma, u(\sigma),(K u)(\sigma)) d \sigma \\
& -\int_{t}^{\infty} U_{Q}(t, \sigma) Q(\sigma) f(\sigma, u(\sigma),(K u)(\sigma)) d \sigma .
\end{aligned}
$$

By Lemma 22, Lemma 23, Lemma 25, and Corollary 19, $\mathscr{F}$ maps $P A A(\mathbb{R}, X, \mu)$ into itself.

For any $u, v \in P A A(\mathbb{R}, X, \mu)$,

$$
\begin{aligned}
& \|(\mathscr{F} u)(t)-(\mathscr{F} v)(t)\| \\
& \leq \int_{-\infty}^{t} \| U(t, \sigma) P(\sigma)[f(\sigma, u(\sigma),(K u)(\sigma)) \\
& -f(\sigma, v(\sigma),(K v)(\sigma))] \| d \sigma \\
& +\int_{t}^{\infty} \| U_{Q}(t, \sigma) Q(\sigma)[f(\sigma, u(\sigma),(K u)(\sigma)) \\
& -f(\sigma, v(\sigma),(K v)(\sigma))] \| d \sigma \\
& \leq L_{f} \int_{-\infty}^{t} M e^{-\delta(t-\sigma)} \\
& \times(\|u(\sigma)-v(\sigma)\|+\|(K u)(\sigma)-(K v)(\sigma)\|) d \sigma \\
& +L_{f} \int_{t}^{\infty} M e^{-\delta(\sigma-t)} \\
& \times(\|u(\sigma)-v(\sigma)\|+\|(K u)(\sigma)-(K v)(\sigma)\|) d \sigma \\
& \leq L_{f}\left(1+\frac{L_{g} C_{k}}{b}\right) \int_{-\infty}^{t} M e^{-\delta(t-\sigma)}\|u(\sigma)-v(\sigma)\| d \sigma \\
& +L_{f}\left(1+\frac{L_{g} C_{k}}{b}\right) \int_{t}^{\infty} M e^{-\delta(\sigma-t)}\|u(\sigma)-v(\sigma)\| d \sigma \\
& \leq \frac{2 M L_{f}\left(b+L_{g} C_{k}\right)}{b \delta}\|u-v\| \text {. }
\end{aligned}
$$

By the Banach contraction mapping principle, $\mathscr{F}$ has a unique fixed point in $P A A(\mathbb{R}, X, \mu)$, which is the unique $P A A$ mild solution to (1). 
Next, consider the following nonautonomous Volterra integrodifferential equations:

$$
u^{\prime}(t)=A(t) u(t)+\int_{-\infty}^{t} k(t-s) u(s) d s+f(t, u(t)),
$$$$
t \in \mathbb{R}
$$

where the linear operators $A(t): D(A(t)) \subset X \rightarrow X$ have a domain $D(A(t))$ not necessarily dense in $X$ and satisfy "Acquistapace-Terreni" conditions and $f$ is a continuous function.

For the pseudo almost automorphy of (40), one has the following.

Theorem 27. Suppose $\left(H_{1}\right),\left(H_{2}\right),\left(H_{3}\right),\left(H_{6}\right)$, and $\left(H_{7_{1}}\right)$ hold, and $f \in P A A(\mathbb{R} \times X, X, \mu)$ satisfies

$\left(H_{9}\right)\|f(t, u)-f(t, v)\| \leq L_{f}\|u-v\|, t \in \mathbb{R}$ and $u, v \in X$, where $L_{f}>0$ is a constant.

Then (40) has a unique mild solution $u \in P A A(\mathbb{R}, X, \mu)$ if $2 M\left(b L_{f}+C_{k}\right)<b \delta$.

Proof. Let

$$
(K u)(t)=\int_{-\infty}^{t} k(t-s) u(s) d s
$$

and define

$$
\begin{aligned}
\Gamma u= & \int_{-\infty}^{t} U(t, \sigma) P(\sigma)((K u)(\sigma)+f(\sigma, u(\sigma))) d \sigma \\
& -\int_{t}^{\infty} U_{Q}(t, \sigma) Q(\sigma)((K u)(\sigma)+f(\sigma, u(\sigma))) d \sigma .
\end{aligned}
$$

Similarly as the proof of Theorem $26, \Gamma$ is well defined and (42) is a mild solution of (40).

For any $u, v \in P A A(\mathbb{R}, X, \mu)$,

$$
\begin{aligned}
& \|(\Gamma u)(t)-(\Gamma v)(t)\| \\
& \leq \int_{-\infty}^{t} M e^{-\delta(t-\sigma)}(\|(K u)(\sigma)-(K v)(\sigma)\| \\
& +\|f(\sigma, u(\sigma))-f(\sigma, v(\sigma))\|) d \sigma \\
& \quad+\int_{t}^{\infty} M e^{-\delta(\sigma-t)}(\|(K u)(\sigma)-(K v)(\sigma)\| \\
& \leq\left(\frac{C_{k}}{b}+L_{f}\right) \int_{-\infty}^{t} M e^{-\delta(t-\sigma)}\|u(\sigma)-v(\sigma)\| d \sigma \\
& \quad+\left(\frac{C_{k}}{b}+L_{f}\right) \int_{t}^{\infty} M e^{-\delta(\sigma-t)}\|u(\sigma)-v(\sigma)\| d \sigma \\
& \leq \frac{2 M\left(b L_{f}+C_{k}\right)}{b \delta}\|u-v\| .
\end{aligned}
$$

By the Banach contraction mapping principle, $\Gamma$ has a unique fixed point in $P A A(\mathbb{R}, X, \mu)$, which is the unique $P A A$ mild solution to (40).

3.2. Pseudo Almost Periodic Perturbation. In this subsection, we investigated the existence and uniqueness of pseudo almost periodic mild solution of (1) and (40). We make the following assumptions:

$$
\begin{aligned}
& \left(H_{7_{2}}\right) R\left(\lambda_{0}, A(\cdot)\right) \in A P(\mathbb{R}, L(X)) \text { for } \lambda_{0} \text { in }\left(H_{1}\right) \\
& \left(H_{8_{2}}\right) g \in P A P(\mathbb{R} \times X, X, \mu) \text { and } f \in P A P(\mathbb{R} \times X \times X, X, \mu)
\end{aligned}
$$
results.

Similarly as the proof of [16], we have the following

Lemma 28. Assume that $h \in A P(\mathbb{R}, X),\left(H_{1}\right),\left(H_{2}\right)$, and $\left(\mathrm{H}_{7_{2}}\right)$ hold; then

$$
\begin{aligned}
(\Pi h)(t)= & \int_{-\infty}^{t} U(t, \sigma) P(\sigma) h(\sigma) d \sigma \\
& -\int_{t}^{\infty} U_{Q}(t, \sigma) Q(\sigma) h(\sigma) d \sigma \in A P(\mathbb{R}, X) .
\end{aligned}
$$

By Lemma 22, Lemma 23, and Lemma 28, similarly as the proof of Theorem 26, Theorem 27, the following results hold.

Theorem 29. Suppose $\left(H_{1}\right)-\left(H_{6}\right),\left(H_{7_{2}}\right)$, and $\left(H_{8_{2}}\right)$ hold; then (1) has a unique mild solution $u \in P A P(\mathbb{R}, X, \mu)$ if $2 M L_{f}(b+$ $\left.L_{g} C_{k}\right)<b \delta$.

Theorem 30. Suppose $\left(H_{1}\right),\left(H_{2}\right),\left(H_{3}\right),\left(H_{6}\right)$, and $\left(H_{7_{2}}\right)$ hold, and $f \in P A P(\mathbb{R} \times X, X, \mu)$ satisfies $\left(H_{9}\right)$, then (40) has a unique mild solution $u \in P A P(\mathbb{R}, X, \mu)$ if $2 M\left(b L_{f}+C_{k}\right)<b \delta$.

3.3. Pseudo Periodic (Antiperiodic) Perturbation. In this subsection, we investigated the existence and uniqueness of pseudo periodic (antiperiodic) mild solution of (1), (40). We make the following assumptions:

$\left(H_{7_{3}}\right)$ there exists $\omega \in \mathbb{R} \backslash\{0\}$ such that $\Gamma(t+\omega, s+\omega)=\Gamma(t, s)$;

$\left(H_{8_{3}}\right) g \in P P_{\omega}(\mathbb{R} \times X, X, \mu), f \in P P_{\omega}(\mathbb{R} \times X \times X, X, \mu)$.

Lemma 31. Assume that $h \in P_{\omega}(\mathbb{R}, X),\left(H_{1}\right),\left(H_{2}\right)$, and $\left(H_{7_{3}}\right)$ hold; then

$$
\begin{aligned}
(\Pi h)(t)= & \int_{-\infty}^{t} U(t, \sigma) P(\sigma) h(\sigma) d \sigma \\
& -\int_{t}^{\infty} U_{Q}(t, \sigma) Q(\sigma) h(\sigma) d \sigma \in P_{\omega}(\mathbb{R}, X) .
\end{aligned}
$$


Proof. Since $h \in P_{\omega}(\mathbb{R}, X)$, then $h(t+\omega)=h(t)$ for $t \in \mathbb{R}$. By $\left(H_{7_{3}}\right)$, one has

$$
\begin{aligned}
& (\Pi h)(t+\omega) \\
& =\int_{-\infty}^{t+\omega} U(t+\omega, \sigma) P(\sigma) h(\sigma) d \sigma \\
& \quad-\int_{t+\omega}^{\infty} U_{Q}(t+\omega, \sigma) Q(\sigma) h(\sigma) d \sigma \\
& =\int_{-\infty}^{t} U(t+\omega, \sigma+\omega) P(\sigma+\omega) h(\sigma+\omega) d \sigma \\
& \quad-\int_{t}^{\infty} U_{Q}(t+\omega, \sigma+\omega) Q(\sigma+\omega) h(\sigma+\omega) d \sigma \\
& =(\Pi h)(t) ;
\end{aligned}
$$

hence, $\Pi h \in P_{\omega}(\mathbb{R}, X)$.

By Lemma 22, Lemma 23, and Lemma 28, similarly as the proof of Theorem 26 and Theorem 27, the following results hold.

Theorem 32. Suppose $\left(H_{1}\right)-\left(H_{6}\right),\left(H_{7_{3}}\right)$, and $\left(H_{8_{3}}\right)$ hold; if $2 M L_{f}\left(b+L_{g} C_{k}\right)<b \delta$, then (1) has a unique mild solution $u \in P P_{\omega}(\mathbb{R}, X, \mu)$.

Theorem 33. Suppose $\left(H_{1}\right),\left(H_{2}\right),\left(H_{3}\right),\left(H_{6}\right)$, and $\left(H_{7_{3}}\right)$ hold, and $f \in P_{\omega}(\mathbb{R} \times X, X, \mu)$ satisfies $\left(H_{9}\right)$; then $(40)$ has a unique mild solution $u \in P P_{\omega}(\mathbb{R}, X, \mu)$ if $2 M\left(b L_{f}+C_{k}\right)<b \delta$.

Remark 34. (i) If $g \in P P_{\text {wap }}(\mathbb{R} \times X, X, \mu)$ and $f \in$ $P P_{\text {wap }}(\mathbb{R} \times X \times X, X, \mu)$ in $\left(H_{8_{3}}\right)$, then Theorem 32 holds for $P P_{\text {wap }}(\mathbb{R}, X, \mu)$; that is, (1) has a unique mild solution $u \in$ $P P_{\omega a p}(\mathbb{R}, X, \mu)$.

(ii) If $f \in P P_{\omega a p}(\mathbb{R} \times X, X, \mu)$, then Theorem 33 holds for $\left.P P_{\text {wap }}(\mathbb{R}, X, \mu)\right)$; that is, (40) has a unique mild solution $u \in$ $P P_{\omega a p}(\mathbb{R}, X, \mu)$.

\section{Example}

In this section, we provide some examples to illustrate our main results.

Example 1. Consider the heat equations with Dirichlet conditions

$$
\begin{gathered}
\frac{\partial u(t, x)}{\partial t}=\frac{\partial^{2} u(t, x)}{\partial x^{2}}+\left(-2+\sin \frac{1}{\cos t+\cos \pi t}\right) u(t, x) \\
+f(t, u(t, x),(K u)(t, x)), \\
(K u)(t, x)=\int_{-\infty}^{t} k(t-s) g(s, u(s, x)) d s, \\
t \in \mathbb{R}, \quad x \in[0, \pi], \\
u(t, 0)=u(t, \pi)=0, \quad t \in \mathbb{R},
\end{gathered}
$$

where $f \in P A A\left(\mathbb{R} \times L^{2}[0, \pi] \times L^{2}[0, \pi], L^{2}[0, \pi], \mu\right), g \in$ $P A A\left(\mathbb{R} \times L^{2}[0, \pi], L^{2}[0, \pi], \mu\right)$, and $\mu \in \mathscr{M}$.
Note that $X=L^{2}[0, \pi]$ is equipped with its natural topology and define

$$
\begin{gathered}
D(A)=\left\{u \in L^{2}[0, \pi]: u^{\prime \prime} \in L^{2}[0, \pi], u(0)=u(\pi)=0\right\}, \\
A u=u^{\prime \prime}-2 u, \quad \forall u \in D(A) .
\end{gathered}
$$

Let $\varphi_{n}(t)=\sqrt{2 / \pi} \sin (n t)$ for all $n \in \mathbb{N}$. It is well known that $A$ is the infinitesimal generator of an analytic semigroup $(T(t))_{t \geq 0}$ on $L^{2}[0, \pi]$ with $\|T(t)\| \leq e^{-3 t}$ for $t \geq 0$ [30]. Moreover,

$$
T(t) \varphi=\sum_{n=1}^{\infty} e^{-\left(n^{2}+2\right) t}\left\langle\varphi, \varphi_{n}\right\rangle \varphi_{n}
$$

for each $\varphi \in L^{2}[0, \pi]$.

Define a family of linear operators $A(t)$ by

$$
\begin{gathered}
D(A(t))=D(A), \\
A(t) \varphi(x)=\left(A+\sin \frac{1}{\cos t+\cos \pi t}\right) \varphi(x), \\
\forall x \in[0, \pi], \quad \varphi \in D(A) .
\end{gathered}
$$

Then the system

$$
\begin{gathered}
u^{\prime}(t)=A(t) u(t), \quad t \geq s, \\
u(s)=\varphi \in L^{2}[0, \pi]
\end{gathered}
$$

has an associated evolution family $(U(t, s))_{t \geq s}$ on $L^{2}[0, \pi]$, which can be explicitly expressed by

$$
U(t, s) \varphi=T(t-s) e^{\int_{s}^{t} \sin (1 /(\cos \tau+\cos \pi \tau)) d \tau} \varphi .
$$

Moreover,

$$
\|U(t, s)\| \leq e^{-2(t-s)} \quad \text { for every } t \geq s .
$$

Note that $\sin (1 /(\cos t+\cos \pi t)) \in A A(\mathbb{R}, \mathbb{R})$, and it is not difficult to verify that $A(t)$ satisfies $\left(H_{1}\right),\left(H_{2}\right),\left(H_{7_{1}}\right)$, and $\left(H_{8_{1}}\right)$ with $M=1$ and $\delta=2$. One can see $[26,31]$ for more details.

Now, the following theorem is an immediate consequence of Theorem 26.

Theorem 35. Under the assumptions $\left(H_{3}\right)-\left(H_{6}\right)$, (47) admits a unique PAA mild solution $u(t) \in P A A\left(\mathbb{R}, L^{2}[0, \pi], \mu\right)$ if $L_{f}\left(b+L_{g} C_{k}\right)<b$.

Example 2. For (40), let $\Omega \subset \mathbb{R}^{n}$ be a bounded domain with $C^{2}$-boundary and $X=L^{2}(\Omega)$. For $t \in \mathbb{R}$, define

$$
A(t)=a(t) \Delta u, \quad u \in D(A(t)):=H_{0}^{1}(\Omega) \cap H^{2}(\Omega),
$$

where $a(\cdot) \in A A(\mathbb{R}, X)$ is Hölder continuous and $\inf _{t \in \mathbb{R}} a(t) \geq a>0$. It is not difficult to see that $\left(H_{1}\right),\left(H_{7_{1}}\right)$ hold and the evolution family $U(t, s)$ generated by $A(t)$ has an exponential dichotomy provided that the Hölder constant 
of $a(\cdot)$ is sufficiently small; hence, $\left(H_{2}\right)$ holds. One can see $[32,33]$ for more details. Suppose further

$$
\begin{array}{r}
k(t-s)=C_{k} e^{-b(t-s)}, \quad \forall t \geq s, \\
f(t, u(x)) \\
=\gamma\left(\sin \frac{1}{2+\cos t+\cos \sqrt{2} t}+\frac{e^{-|t|}}{(1+|t|)^{2}}\right) \cos (u(x)), \\
\forall t \in \mathbb{R}, \quad u \in X, \quad x \in \Omega,
\end{array}
$$

where $C_{k}, b$, and $\gamma$ are positive constants; then $f \in P A A(\mathbb{R}$, $X, \mu) ; \mu$ is the Lebesgue measure, so $\left(H_{3}\right),\left(H_{6}\right)$ hold. Moreover,

$$
\|f(t, u)-f(t, v)\| \leq 2 \gamma\|u-v\|, \quad \forall t \in \mathbb{R}, u \in X, x \in \Omega,
$$

which implies that $\left(H_{9}\right)$ holds. Therefore, $(40)$ has a unique mild solution $u \in P A A(\mathbb{R}, X, \mu)$ if $\gamma$ is sufficiently small by Theorem 27.

\section{Conflict of Interests}

The author declares that there is no conflict of interests regarding the publication of this paper.

\section{Acknowledgment}

This research was supported by Zhejiang Provincial Natural Science Foundation of China under Grant no. LQ13A010015.

\section{References}

[1] N. S. Al-Islam, S. M. Alsulami, and T. Diagana, "Existence of weighted pseudo anti-periodic solutions to some nonautonomous differential equations," Applied Mathematics and Computation, vol. 218, no. 11, pp. 6536-6548, 2012.

[2] J. Blot, G. M. Mophou, G. M. N’Guérékata, and D. Pennequin, "Weighted pseudo almost automorphic functions and applications to abstract differential equations," Nonlinear Analysis, Theory, Methods and Applications, vol. 71, no. 3-4, pp. 903-909, 2009.

[3] T. Diagana, "Weighted pseudo almost periodic functions and applications," Comptes Rendus Mathematique, vol. 343, no. 10, pp. 643-646, 2006.

[4] C. Lizama and G. M. N'Guérékata, "Bounded mild solutions for semilinear integro differential equations in Banach spaces," Integral Equations and Operator Theory, vol. 68, no. 2, pp. 207227,2010

[5] Z. Xia, "Weighted Stepanov-like pseudoperiodicity and applications," Abstract and Applied Analysis, vol. 2014, Article ID 980869, 14 pages, 2014.

[6] J. Blot, P. Cieutat, and K. Ezzinbi, "New approach for weighted pseudo-almost periodic functions under the light of measure theory, basic results and applications," Applicable Analysis, vol. 92, no. 3, pp. 493-526, 2013.
[7] J. Blot, P. Cieutat, and K. Ezzinbi, "Measure theory and pseudo almost automorphic functions: new developments and applications," Nonlinear Analysis: Theory, Methods \& Applications, vol. 75, no. 4, pp. 2426-2447, 2012.

[8] S. Abbas, "Pseudo almost automorphic solutions of some nonlinear integro-differential equations," Computers and Mathematics with Applications, vol. 62, no. 5, pp. 2259-2272, 2011.

[9] H. S. Ding, J. Liang, and T. J. Xiao, "Pseudo almost periodic solutions to integro-differential equations of heat conduction in materials with memory," Nonlinear Analysis: Real World Applications, vol. 13, no. 6, pp. 2659-2670, 2012.

[10] E. Hernández and J. P. C. Santos, "Asymptotically almost periodic and almost periodic solutions for a class of partial integrodifferential equations," Electronic Journal of Differential Equations, vol. 2006, no. 38, pp. 1-8, 2006.

[11] C. Lizama and R. Ponce, "Bounded solutions to a class of semilinear integro-differential equations in Banach spaces," Nonlinear Analysis: Theory, Methods \& Applications, vol. 74, no. 10, pp. 3397-3406, 2011.

[12] I. Mishra and D. Bahuguna, "Weighted pseudo almost automorphic solution of an integro-differential equation, with weighted Stepanov-like pseudo almost automorphic forcing term," Applied Mathematics and Computation, vol. 219, no. 10, pp. 5345-5355, 2013.

[13] Z. N. Xia, "Weighted pseudo almost automorphic solutions of hyperbolic semilinear integro-differential equations," Nonlinear Analysis: Theory, Methods \& Applications, vol. 95, pp. 50-65, 2014.

[14] T. Diagana, "Existence results for some nonautonomous integro-differential equations," http://arxiv.org/abs/1402.5638vl.

[15] T. Diagana and G. M. N'Guérékata, "Pseudo almost periodic mild solutions to hyperbolic evolution equations in intermediate Banach spaces," Applicable Analysis, vol. 85, no. 6-7, pp. 769780, 2006.

[16] H. S. Ding, J. Liang, G. M. N’Guérékata, and T. J. Xiao, “Pseudo almost periodicity to some nonautonomous semilinear evolution equations," Mathematical and Computer Modelling, vol. 45, pp. 579-584, 2007.

[17] K. Engel and R. Nagel, One-Parameter Semigroups for Linear Evolution Equations, vol. 194 of Graduate Texts in Mathematics, Springer, New York, NY, USA, 2000.

[18] A. M. Fink, Almost Periodic Differential Equations, Springer, New York, NY, USA, 1974.

[19] S. Bochner, "A new approach to almost periodicity," Proceedings of the National Academy of Sciences of the United States of America, vol. 48, pp. 2039-2043, 1962.

[20] C. Zhang, Pseudo almost periodic functions and their applications [Ph.D. thesis], University of Western Ontario, 1992.

[21] G. M. N'Guérékata, Topics in Almost Automorphy, Springer, New York, NY, USA, 2005.

[22] M. Adimy, K. Ezzinbi, and C. Marquet, "Ergodic and weighted pseudo-almost periodic solutions for partial functional differential equations in fading memory spaces," Journal of Applied Mathematics and Computing, vol. 44, no. 1-2, pp. 147-165, 2014.

[23] P. Acquistapace and B. Terreni, "A unified approach to abstract linear nonautonomous parabolic equations," Rendiconti del Seminario Matematico della Università di Padova, vol. 78, pp. 47-107, 1987.

[24] P. Acquistapace, "Evolution operators and strong solutions of abstract linear parabolic equations," Differential and Integral Equations, vol. 1, no. 4, pp. 433-457, 1988. 
[25] Z. Hu and Z. Jin, "Almost automorphic mild solutions to neutral parabolic nonautonomous evolution equations with nondense domain," Discrete Dynamics in Nature and Society, vol. 2013, Article ID 183420, 10 pages, 2013.

[26] Z. R. Hu and Z. Jin, "Stepanov-like pseudo almost automorphic mild solutions to nonautonomous evolution equations," Nonlinear Analysis, Theory, Methods and Applications, vol. 71, no. 5-6, pp. 2349-2360, 2009.

[27] T. Diagana, "Existence and uniqueness of pseudo-almost periodic solutions to some classes of partial evolution equations," Nonlinear Analysis, Theory, Methods and Applications, vol. 66, no. 2, pp. 384-395, 2007.

[28] G. M. N’Guérékata, "Existence and uniqueness of almost automorphic mild solutions to some semilinear abstract differential equations," Semigroup Forum, vol. 69, no. 1, pp. 80-86, 2004.

[29] T. Xiao, X. Zhu, and J. Liang, "Pseudo-almost automorphic mild solutions to nonautonomous differential equations and applications," Nonlinear Analysis: Theory, Methods and Applications, vol. 70, no. 11, pp. 4079-4085, 2009.

[30] A. Lunardi, Analytic Semigroups and Optimal Regularity in Parabolic Problems, vol. 16 of Progress in Nonlinear Differential Equations and Their Applications, Birkhäuser, Basel, Switzerland, 1995.

[31] T. Diagana, "Stepanov-like pseudo-almost periodicity and its applications to some nonautonomous differential equations," Nonlinear Analysis: Theory, Methods and Applications, vol. 69, no. 12, pp. 4277-4285, 2008.

[32] T. Diagana, "Existence of weighted pseudo-almost periodic solutions to some classes of nonautonomous partial evolution equations," Nonlinear Analysis: Theory, Methods \& Applications, vol. 74, no. 2, pp. 600-615, 2011.

[33] H. S. Ding, J. Liang, G. M. N’Guérékata, and T. Xiao, "Pseudoalmost periodicity of some nonautonomous evolution equations with delay," Nonlinear Analysis: Theory, Methods and Applications, vol. 67, no. 5, pp. 1412-1418, 2007. 


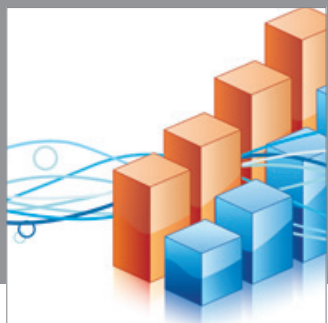

Advances in

Operations Research

mansans

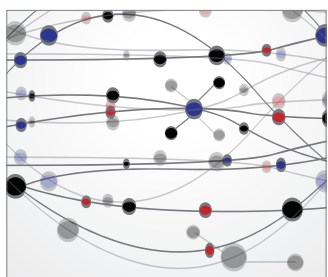

The Scientific World Journal
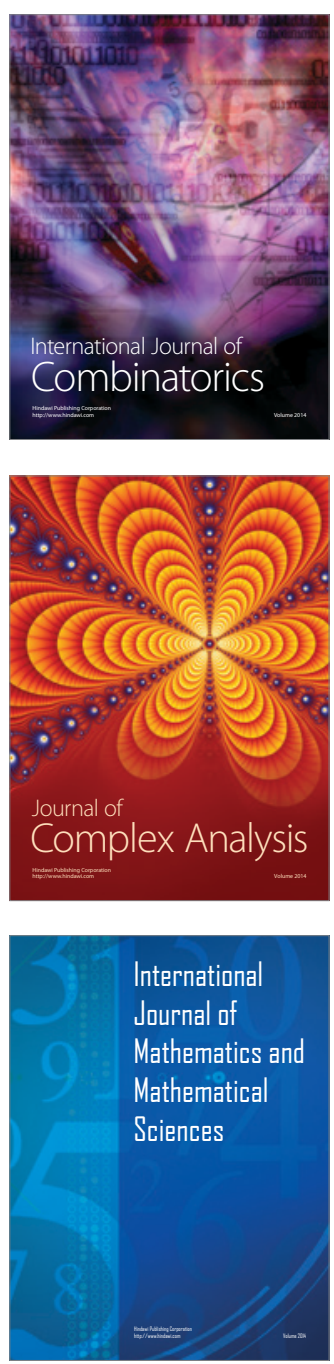
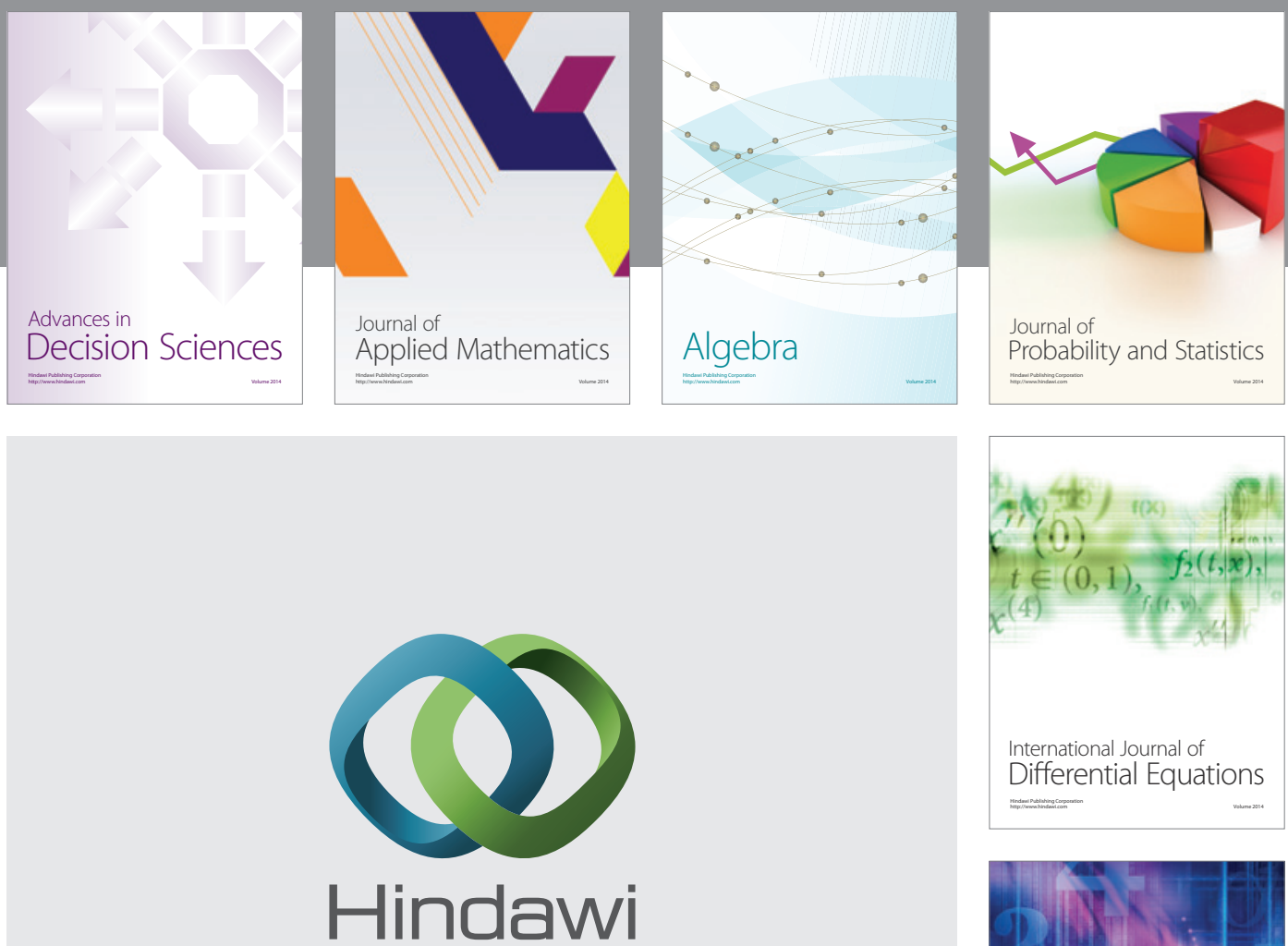

Submit your manuscripts at http://www.hindawi.com
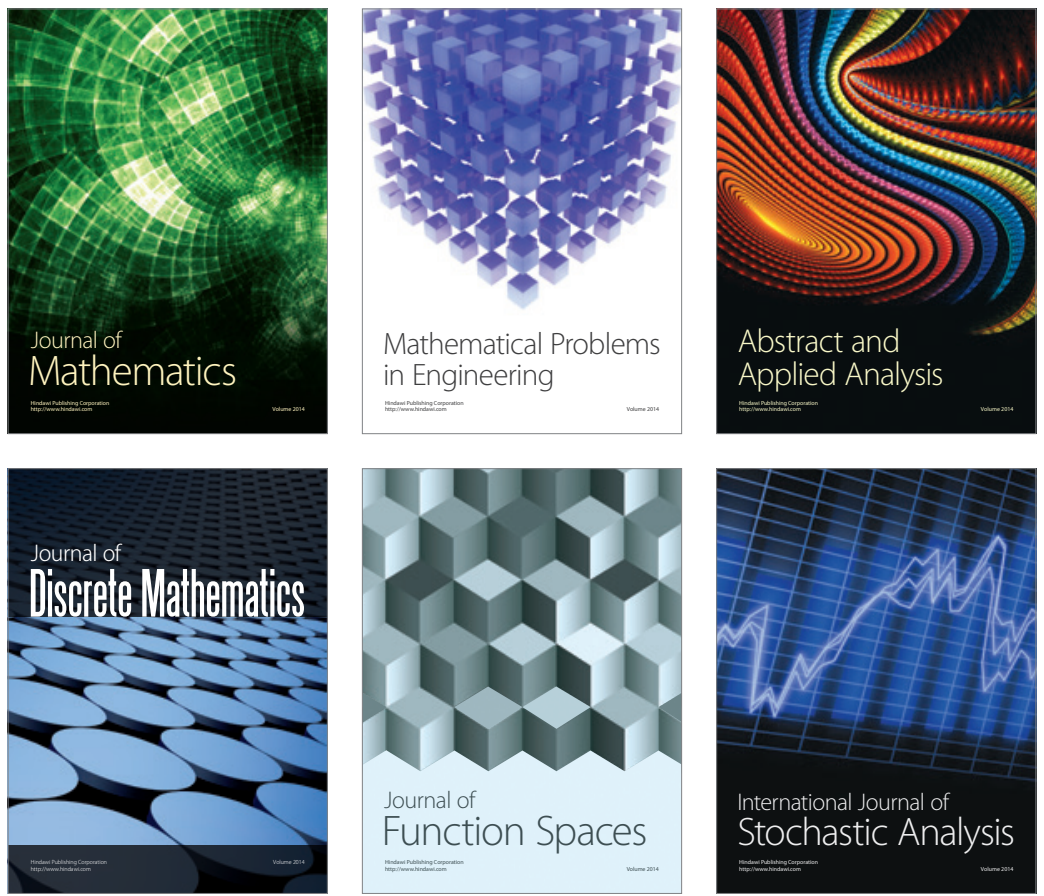

Journal of

Function Spaces

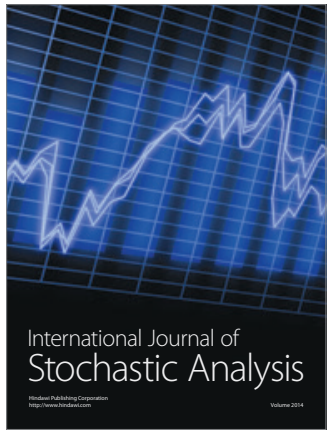

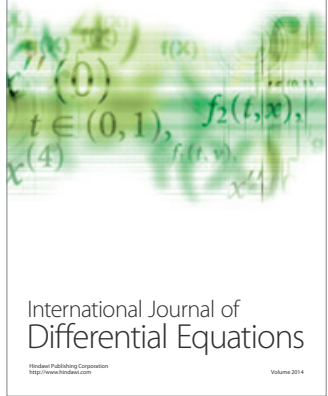
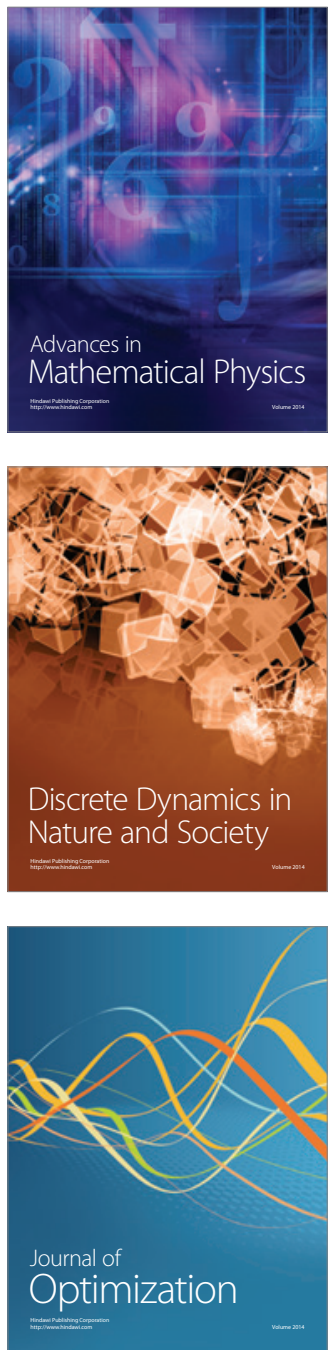